\title{
Soft Computing decision support for a steel sheet incremental cold shaping process *
}

\author{
José R. Villar ${ }^{1 \dagger}$, Javier Sedano ${ }^{2}$, Emilio Corchado ${ }^{3}$, and Laura Puigpinós ${ }^{4}$ \\ 1 University of Oviedo, Gijón Asturias 33204, Spain \\ villarjoseduniovi.es, \\ 2 Instituto Tecnoló de Castilla y León, Poligono Industrial de Villalonquejar, Burgos (SPAIN), \\ javier.sedanoditcl.es, \\ 3 Computer Science and Automatica Department with the University of Salamanca, Plaza de la \\ Merced s/n 37008 Salamanca (SPAIN) \\ escorchado@usal.es \\ 4 Fundación Privada Ascamm, Avda. Universitat Autònoma, 2308290 Cerdanyola del Vallés
}

(SPAIN) lpuigpinos@ascamm.com

\begin{abstract}
It is known that the complexity inherited in most of the new real world problems, for example, the cold rolled steel industrial process, increases as the computer capacity does. Higher performance requirements with a lower amount of data samples are needed due to the costs of generating new instances, specially in those processes where new technologies arise. This study is focused on the analysis and design of a novel decision support system for an incremental steel cold shaping process, where there is a lack of knowledge of which operating conditions are suitable for obtaining high quality results. The most suitable features have been found using a wrapper feature selection method, in which genetic algorithms and neural networks are hybridized. Some facts concerning the enhanced experimentation needed and the improvements in the algorithm are drawn.
\end{abstract}

Keywords: Wrapper Feature Selection, Genetic Algorithms, Neural Networks, Support Vector Machines, Incremental Cold Shaping

\section{Introduction}

Over recent years there has been a high increase in the use of artificial intelligence and Soft Computing methods to solve real world problems. Many different applications have been reported: the use of Exploratory Projection Pursuit (EPS) [3] and ARMAX for modelling the manufacture of steel components [4], EPS and neural networks for determining the operation conditions in face milling operations and in pneumatic drilling process [10], genetic algorithms and programming for trading rule extraction [5] and low quality data in lighting control systems [14], feature selection and association rule

${ }^{*}$ This research has been partially funded by the projects TIN2008-06681-C06-04, TIN201021272-C02-01 and PID 560300-2009-11 of the Spanish Ministry of Science and Innovation, by the Junta de Castilla y León CCTT/10/BU/0002 project, by the FICYT project CN-08-028-IE0760 and by the ITCL project CONSOCO.

${ }^{\dagger}$ Corresponding author 
discovery in high dimensional spaces [13] or neural networks (NN) and EPS in building energy efficiency [11,12].

It is known that the complexity inherited in most of the new real world problems, as for example, the steel cold shaping industrial process, is increasing as the computer capacity does. Higher performance requirements with a lower amount of data samples is needed due to the costs of generating new instances, specially in those processes where new technologies arise.

In this sense, the steel cold shaping is a relatively new technology in the production of low quantity lots steel pieces, which represents an effervescent area. Neural networks have been used to find relationships between the mechanical properties of the cold-rolled sheets of interstitial free and the chemical composition of the steel and the rolling and the batch annealing parameters [9]. Neural networks have been applied for identification of the parameters for operating conditions [18, 19]. Up to our knowledge, no specific study has been published in steel iterative cold shaping.

This study focuses on determining the main parameters in an steel sheet incremental cold shaping. The main objective is to find the most relevant feature subset; the second objective is to obtain a decision support system in the operating conditions design, so the costs of producing such lots with a low amount of pieces is reduced. The next Section is concerned with the problem description. In Section 3 the algorithm used is detailed, while Sect. 4 deals with the experiments carried out and the results obtained. Finally, conclusions and future work are drawn.

\section{Steel incremental cold shaping}

The metal incremental cold shaping is based on the concept of incremental deformation. This technology allows the manufacturing of pieces of metal sheet through the iteration of small sequential deformation stages until the desired shape is achieved and avoiding the axis-symmetric restrictions due to incremental rotatory deformation. Comparing incremental cold shaping with traditional deformation technologies it can be said that the former reduces the cost of specific machine tools and the manufacturing costs dramatically.

This type of technology has evolved from the well-known Rapid Manufacturing, allowing to generate pieces with complex geometries in a wide spread of materials without the need of frameworks or specific tools.

The main part of cold shaping has been controlled using numerical controlled tools in order to reduce as much as possible the fast, reliable, and costless manufacturing of lots with and small amount of metal pieces and prototypes.

The process of cold shaping starts with the design of a geometric shape in a 3D CAD file. This file should include as many layers as desired, each layer represents the bounds to be reached in each deforming step and are piled vertically. Consequently, the piece should be generated using sequential and incremental layers, each one at a different depth and constraint within the defined bounds.

Plenty of parameters have to be fixed to manufacture a metal piece, the force in each of the three dimensions to be develop by the deforming head, the speed change, the trajectory of the head, the surface roughness, the sheet pressure stress, the incremental 
step between layers, the number of steps or stages, the attack angle, the angle variation, the depth variation, etc. In Table 1, the range of the values of several of the input features is shown.

Table 1. Typical values of several of the variables involved in steel sheet incremental cold shaping.

\begin{tabular}{|l|c|c|}
\hline Variable & Units & Range of values \\
\hline Sheet pressure stress & $\mathrm{MPa}$ & {$[75,180]$} \\
Surface roughness about the tool & $\mathrm{mm}$ & {$[0.1,0.5]$} \\
Speed change & $\mathrm{mm} / \mathrm{m}$ & {$[800,6000]$} \\
Force to be applied in the x-axis for shaping & $\mathrm{N}$ & {$[250,500]$} \\
Force to be applied in the y-axis for shaping & $\mathrm{N}$ & {$[250,500]$} \\
Force to be applied in the z-axis for shaping & $\mathrm{N}$ & {$[500,1000]$} \\
Depth variation & $\mathrm{mm}$ & {$[0,9]$} \\
Angle variation & degrees & {$[3,15]$} \\
Number of incremental layers & & {$[3,6]$} \\
Incremental step between layers & $\mathrm{mm}$ & {$[0.3,0.8]$} \\
Minimum thickness & $\mathrm{mm}$ & {$[0.28,0.40]$} \\
Maximum depth achieved & $\mathrm{mm}$ & {$[-16.4,-39]$} \\
\hline
\end{tabular}

\subsection{The problem definition}

The first aim of this study is to evaluate if it is possible to model the operating conditions so the suitability of the experiment could be established, in other words, to analyse whether the operating conditions would generate a faulty piece or not while the most relevant features involved are to be selected.

The second aim is to model the maximum suitable depth that can be achieved with the given operating conditions. As in the former problem, the best feature subset is also required.

Therefore, there are two problems to solve, both including a feature selection process and a modelling process. While the former is a two-class problem, the second is a regression problem.

\section{$3 \quad$ Feature Selection and neural networks}

In order to obtain a suitable feature subset some requirements are needed. As there are integer features, nominal features and real valued features, the algorithm should deal with any kind of data. Therefore, the same approach should be valid for the both subproblems, the two-class problem and the maximum depth. Besides, not only the best feature subset for each problem but also the best model are desired, a classifier in the former case and a regression model in he latter.

It is known that for this kind of problems the wrapper approach for feature selection performs better than filter solutions $[2,16]$. These studies proposed wrapper feature 
selection methods using genetic algorithms (GA) [2] for dealing with the feature subset selection, that is, each individual is a feature subset. To evaluate individuals a modeling technique has been applied: the former proposed a lazy learning model as the K-Nearest Neighbour (KNN), the latter made use of a neural network (NN) method that iteratively fix the number of hidden neurons.

Different approaches as to how the NN is learnt have been studied. In [1] a GA approach to fingerprint feature selection is proposed and selected features are supplied as input to $\mathrm{NN}$ for fingerprint recognition, while in [15] a similar approach has been applied to automatic digital modulation recognition. Moreover, this type of approach has been reported to perform bettern that using statistical models [17]. Despite this, Support Vector Machines (SVM) have been also used in conjunction with evolutionary feature selection to reduce the input space dimensionality $[6,7]$.

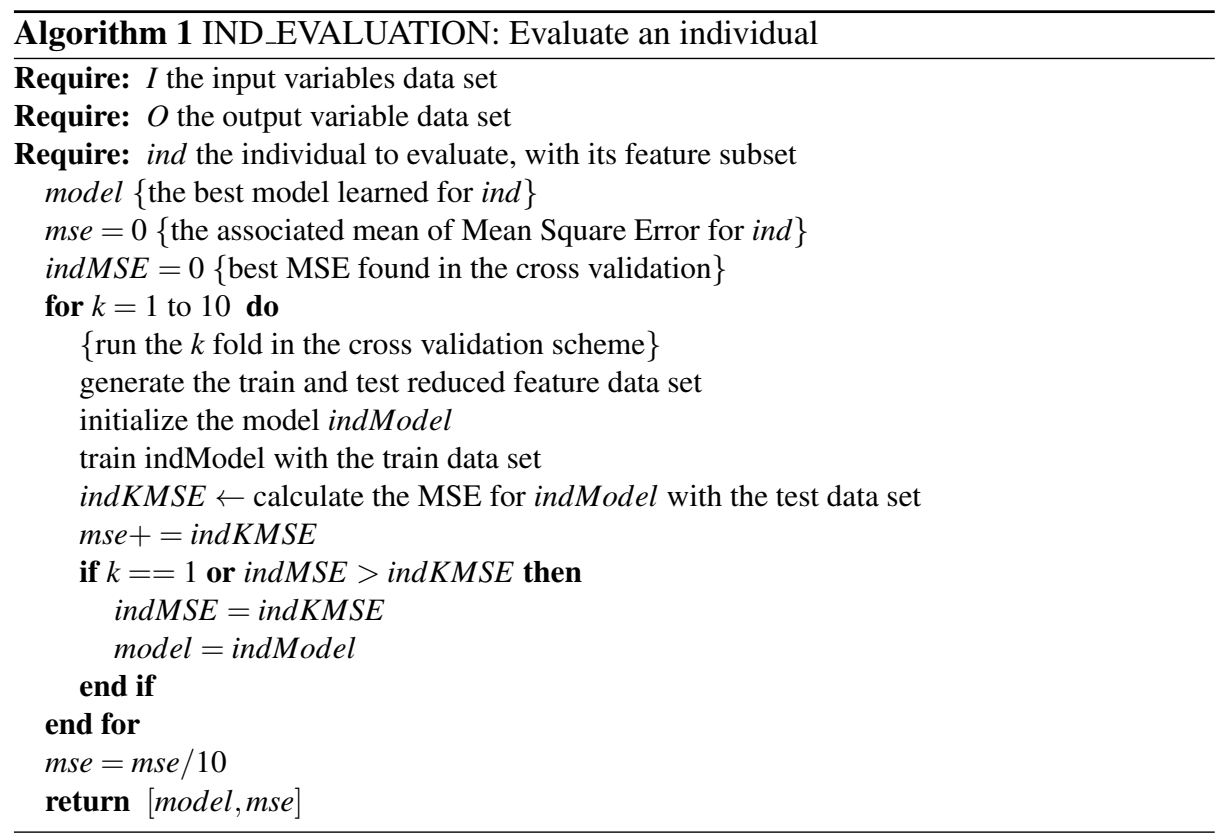

In this study we adopt two different solutions depending whether we are dealing with the two-class or the maximum depth problem. An hybridized method of GA evolving the feature subsets and a SVM classifier is chosen in the former case, while in the latter an hybridized method of GA evolving the feature subsets and a NN for modeling the desired output is used. TIn both modelling and feature selection problems the GA is an steady state approach with the percentage of elite individuals to be defined as a parameter. The algorithm is outlined in Algorithms [1,2].

The typical steady state GA parameters, like the crossover and mutation probabilities, the number of generations, the population size and the elite population size, are all of them given for each experiment. The individual representation is the string of indexes 





of the chosen feature subset. The tournament selection is implemented and one point crossover is used. After each genetic operation the validity of the off-prints is analysis: repeated features indexes are erased and random indexes are introduced to fill the individual feature subset.

Third order polynomials are used as kernel functions for the SVM. The number of hidden nodes in the $\mathrm{NN}$ is set as a parameter. The NN models are generated randomly and trained. In all cases, 10-fold cross validation is used, and the mean value of the mean squared error in each fold is the fitness of an individual.

\section{Experiments and results}

Generating data set samples is costly as each one of the samples needs a real case to be carried out, that is, a sheet of steel has to be cold shaped; consequently, the smaller the number of experiments, the lower the cost and the smaller the data set size.

The data set comprises 19 samples, each one with the whole set of parameters values. Once the piece is processed as the corresponding sample establishes, then it is manually classified as $\{$ GOOD, BAD $\}$ according to the deformation or the quality faults that could appear in the piece. Besides, the maximum depth in each case is also measured. These two latter values are appended to each sample as the output variables.

As SVM and NN are to be used in the modelling part of the feature selection GA method, then the data set is normalized with means 0 and deviations 1 .

In the experimentation, the GA parameters have been fixed as follows: 50 individuals in the population, 100 of generations, the probability of crossover equals to 0.75 , while the mutation probability is 0.25 . An steady state GA evolutionary scheme is used, with a number of 5 elite individuals that will be kept in the next generation.

The size of the feature subset has been fixed to three. The SVM kernel function is fixed as third order polynomials and the feed forward back-propagation NNs includes 6 neurons in the hidden layer. The parameters of the SVM and the NN have been kept constant during the feature selection and model learning.

As stated in the previous section, the 10-fold cross validation schema is carried out. Only the validation results are used to compare individuals. For the two-class problem the mean of the classification errors among the folds is used to evaluate the models and the feature subsets. For the maximum depth estimation, each feature subset and its corresponding model are evaluated with the mean of the mean squared error on each fold.

In the case of the two-class problem, the best feature subset found includes the step increment, the angle variation and the variation in depth, with a mean classification error of 0.1 . For the second problem, the best feature subset found includes the variables step increment, the number of stages and variation in depth, with a mean error of 0.0096 .

It is worth mentioning that the reduced number of data samples induces relatively high error values as the test data set includes only one or two examples. More accuracy should be obtained if a bigger number of samples is given. However, the cost of the data gathering increases considerably; this dilemma should be evaluated. 
Moreover, the algorithms do not include local optimization of the models parameters. So it is possible that better performance of the models and the feature selection process could be more affordable if such local optimization were implemented.

Finally, the maximum depth have been found regardless of the two-class problem, which was not the expected result in the expert opinion. It is though that the above mentioned local optimization of the models parameters should improve the performance and the experts confidence in the results.

\section{Conclusions and future work}

This study introduces a feature selection method for choosing the best feature subset in a steel sheets cold shaping process divided in a two-class problem and a maximum depth estimation problem. Moreover, a genetic algorithm is hybridized, on the one hand, for the first case, with a support vector machine model to choose the best feature subset and on the other hand, for the second case, with a feed foward back-propagation neural network .

From the experimentation the best feature subset has been found for both problems, and some relevant facts have arisen. Firstly, the data set size should be increased in order to obtain better models fitness values. Secondly, local optimization for the models parameters should improve the obtained results. Finally, it could be desirable that the optimum number of features should be dynamically fixed, which represents an improvement in the individual representation and is left for future work. Future work also includes evaluating the approach in more detail and including a comparison to the related work.

\section{References}

1. Altun, A. A., Allahverdi, N.: Neural Network Based Recognition by Using Genetic Algorithm for Feature Selection of Enhanced Fingerprints. ICANNGA '07 Proceedings of the 8th international conference on Adaptive and Natural Computing Algorithms, Part II. Springer-Verlag Berlin, Heidelberg (2007)

2. Casillas, J., Cordón, O., del Jesus, M. J., Herrera, F. (2001) Genetic Feature Selection in a Fuzzy Rule-Based Classification System Learning Process. Information Sciences 136:1-4, $135-157$

3. Corchado, E., MacDonald, D., Fyfe, C.: Maximum and Minimum Likelihood Hebbian Learning for Exploratory Projection Pursuit. Data Min. Knowl. Discov. 8(3): 203-225 (2004)

4. Corchado, E., Sedano, J., Curiel, L., Villar, J. R.: Optimizing the operating conditions in a high precision industrial process using soft computing techniques. Expert Systems, in press (2011)

5. de la Cal, E., Fernández, E. M., Quiroga, R., Villar, J., Sedano, J. (2010) Scalability of a Methodology for Generating Technical Trading Rules with GAPs Based on Risk-Return Adjustment and Incremental Training. LNCS 6077, 143-150

6. Fung, G. M., Mangasarian, O.L. (2004) A Feature Selection Newton Method for Support Vector Machine Classification. Computational Optimization and Applications 28:2, 185-202

7. Huanga, C.-L., Wang, C.-J. (2006) A GA-based feature selection and parameters optimizationfor support vector machines. Expert Systems with Applications 31:2, 231-240 
8. The MathWorks (2011) MATLAB - The Language Of Technical Computing http://www.mathworks.com/products/matlab/

9. Mohanty, I., Datta, S., Bhattacharjeeb, D. (2009) Composition-Processing-Property Correlation of Cold-Rolled IF Steel Sheets Using Neural Network. Materials and Manufacturing Processes 24:1, 100-105

10. Sedano, J., Corchado, E., Curiel, L., Villar, J., Bravo, P. (2008) The Application of a TwoStep AI Model to an Automated Pneumatic Drilling Process. Int. J. of Comp. Mat. 86:10-11, 1769-1777

11. Sedano, J., Curiel, L., Corchado, E., de la Cal, E., Villar, J. R. (2009) A Soft Computing Based Method for Detecting Lifetime Building Thermal Insulation Failures. Int. Comp.-Aided Eng. 17:2, 103-115

12. Sedano, J., Villar, J. R., Curiel, L., de la Cal, E., Corchado, E. (2009) Improving Energy Efficiency in Buildings Using Machine Intelligence. 10th Int. Conf. on Intelligent Data Engineering and Automated Learning (IDEAL 2009), LNCS 5788, 773-782

13. Villar, J. R., Suárez, M. R., Sedano, J., Mateos, F. (2009) Unsupervised Feature Selection in High Dimensional Spaces and Uncertainty. 4th International Workshop on Hybrid Artificial Intelligence Systems, LNCS 5572, 565-572

14. Villar, J. R., Berzosa, A., de la Cal, E., Sedano, J., García-Tamargo, M. (2011) Multiobjective Simulated Annealing in Genetic Algorithm and Programming learning with low quality data. In publication for Neural Computing

15. Wong, M. L. D., Nandi, A. K. (2004) Automatic digital modulation recognition using artificial neural network and genetic algorithm. Signal Proc. 84:2 351-365

16. Yang, J., Honavar, V. (1998) Feature Subset Selection Using a Genetic Algorithm. IEEE Intelligent Systems 13:2, 44-49.

17. Zhang, P., Verma, B., Kumar, K. (2005) Neural vs. statistical classifier in conjunction with genetic algorithm based feature selection. Pat. Recog. Letters 28:7 909-919

18. Zhao, J., Cao, H. Q., Ma, L.X., Wang, F. Q., Li, S. B. (2005) Study on intelligent control technology for the deep drawing of an axi-symmetric shell part. J. of Materials Processing Tech. 151:1-3, 98-104

19. Zhao, J., Wang, F. (2005) Parameter identification by neural network for intelligent deep drawing of axisymmetric workpieces. J. of Materials Processing Tech. 166:3, 387-391 